\title{
Applications of Internet of Things in the Facility Agriculture
}

\author{
Linli Zhou, Liangtu Song, Chengjun Xie, and Jie Zhang \\ Institute of Intelligent Machines, Chinese Academy of Sciences \\ 230031, Hefei China \\ Linlizhou@iim.ac.cn
}

\begin{abstract}
It is a trend to use information technology to lead the development of modern agriculture. The IntelliSense Internet of Things will be an important support for intensive, high-yield, high-quality, efficient, ecological security agriculture. In this paper, we give solutions and key technologies of facilities agriculture based on the Internet of Things technology. On this basis it designs and implements facility cultivation greenhouses. Practice has proved that the Internet of Things is the development of modern agriculture productivity. It has an important significance in raising the level of agricultural development, improving the overall efficiency of agriculture, promoting the upgrade of modern agricultural transformation.
\end{abstract}

Keywords: Internet of Things, agricultural facilities, wireless sensing, intelligent control.

\section{$1 \quad$ Introduction}

Facilities agriculture is a new agricultural industry which has a high degree of intensification. It is an important part of modern agriculture. In recent years, the technology of Internet of Things which continues to evolve and mature has injected new vitality into the development of agricultural facilities. IntelliSense chips, mobile embedded systems such as the Internet of Things technology in modern agriculture are gradually widened. Using wireless sensor networks can reduce the impact of human consumption and the farmland environment. Extensive use of automation, intelligent remote-controlled production equipment can obtain accurate crop and crop information. Through these, people who stay at home can monitor a variety of field information. This can achieve the scientific cultivation, scientific monitoring and production management and promote modern agriculture development pattern.

\section{The Design of facility Agricultural Based on Internet of Things}

The facility agricultural system based on the Internet of Things technology is divided into three levels: the perception layer, transport layer and application layer. Perception 
layer is mainly responsible for data-aware acquisition; the transport layer is mainly responsible for the perception of data transmission; application layer is mainly responsible for sensing data analysis, statistics, and early warning, automatic control and scientific decision-making. Fig.1 shows the overall architecture diagram of the facility agriculture.

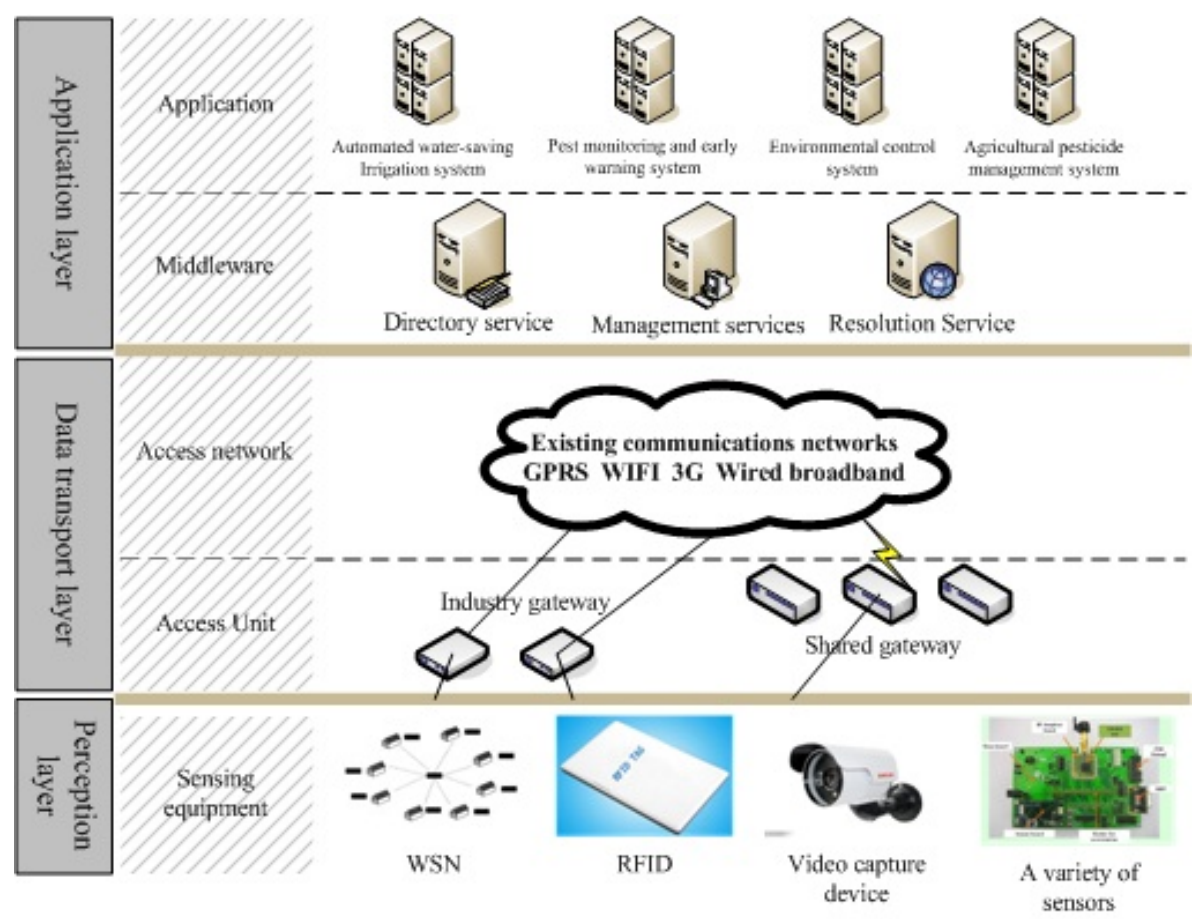

Fig. 1. The architecture diagram of facility agriculture

\subsection{Data Perception and Acquisition}

Information sensing technology of facility agricultural is stressed that the perception of intelligence and automation of information. Perception information should be processed intelligently and transported wirelessly. The Facility agricultural information has high demand to the perception and perception equipment. They must enable quick determination of the object, but also need to have a dynamic, continuous determination of the characteristics of the wireless sensing and wireless transmission. The sensing devices need to have the characteristics of micro, reliability, energysaving, environmental adaptability, low cost and intelligent. Information-aware technology can be used for soil parameters, the continuous monitoring of plant nutrients, and the fast dynamic of the environmental parameters. 


\subsection{Wireless Sensor Networks}

Wireless sensor networks include wireless sensor nodes, sink nodes, the routing node, the central base station, network data server and remote access node. Sensor node is responsible for the collection, a variety of soil and environmental parameters of the storage location. These parameters include air temperature, humidity, sunshine intensity, soil temperature, moisture, $\mathrm{PH}$ value. Sensor node sends data to the sink node through a variety of means of communication. Aggregation node is responsible for data collection, filtering and storage in wireless sensor clusters, and to communicate with the wireless routing nodes forward data timely.

\subsection{Application of Decision-Making Platform}

Application of decision-making platform includes the intelligent processing of information, cloud storage and application. Facility agriculture in the prenatal, delivery, postpartum industrial chain, relying on the cloud application service platform for the base production, the platform needs to be developed greenhouse environment control systems, fertilizer drug control management system, pest and disease monitoring and early warning systems, agricultural quality of the distribution process safety monitoring system. For government decision-making, technical guidance to farmers, public consumption and other aspects of the platform required the development and application of centralized display system, pests and diseases of the joint prevention and control command and decision system, remote expert guidance systems, facilities, vegetables, green resume system, etc..

\section{The Key Technologies of Facility Agriculture}

\subsection{RFID Technology}

Facility agricultural system has a large number of device management and remote control, you need to effectively identify and distinguish the equipment. Uniquely identify and read by the RFID technology, together with the GPS positioning method can control and manage the equipment location of Things, so as to achieve the purpose of the overall perception and intelligent control. There are more RFID products, but only fewer products for facility agricultural use.

\subsection{Sensor Technology}

The sensors are the eyes and ears of the information age; the sensor has a wide application in facility agriculture. The sensors can monitor the environmental conditions of the crop and the dynamic data which got through the facilities intervention. Commonly used sensors of agricultural facility include light sensors, temperature and humidity sensors, pressure-sensitive (fluid) sensor, CO2 sensor, the value of the sensor, as well as plant growth characteristics of sensors and other sensors. 


\subsection{Automation Technology}

Facility agricultural needs automation technology for environmental regulation. According to the set of environmental conditions the system get control of greenhouse fertilization, irrigation, opening and closing doors and windows, lifting moderate light adjustments and others through the controlling system and execution system. The nature of the automation technology is mechatronics, controller and executive body is the core of the automation technology. Automation technology is the traditional industries; the development is already quite mature. Equipment intelligent control has been achieved. Development of automation technology which greatly improves labor productivity, and contributed to improve the system closedloop control of the Internet of Things has been developing rapidly in a wide range of needs.

\subsection{Wireless Data Transmission Technology}

With the development of information technology, the wireless transmission technology has been developing rapidly. ZigBee technology occupies a dominant position. ZigBee technology is a unified standard, short-range wireless communications technology, with the characteristics of low power, low cost and versatility. WIFI has a high transmission rate, transmission distance and cover a wide range of features; it is widely used in data transmission in computer networks and mobile communications. GPRS and $3 \mathrm{G}$ digital communications technology has begun to be applied in the field of agricultural facility. It has a stable network; the terminal price is low and the maturity of the technology and other advantages.

\subsection{Intelligent Information Processing Technology}

Intelligent information processing system has been used in the field of agricultural facilities, real-time control of the parameters of the environmental temperature and humidity, light, fertilizer and liquid level. The development of cloud computing has injected new elements for facilities management in agriculture.

\section{Implementation of the Facility Agriculture Project}

On the basis of the research of facility agricultural structure and key technologies, sensing technology, infinite communication technology, computer network technology, agricultural resources, database technology, Internet of Things technology were used to build management technology platform for facility agriculture. It develops intelligent, accurate scientific production management for the majority of cooperatives, grower, facilities and agricultural enterprises and other users. Developed science intelligent precision operation scheme can make reasonable use of resources; improve product yield and efficiency; and improve the quality of agricultural products so as to enhance the market competitiveness of industry standards and product. Fig.2 shows the function and structure of facility agriculture. 


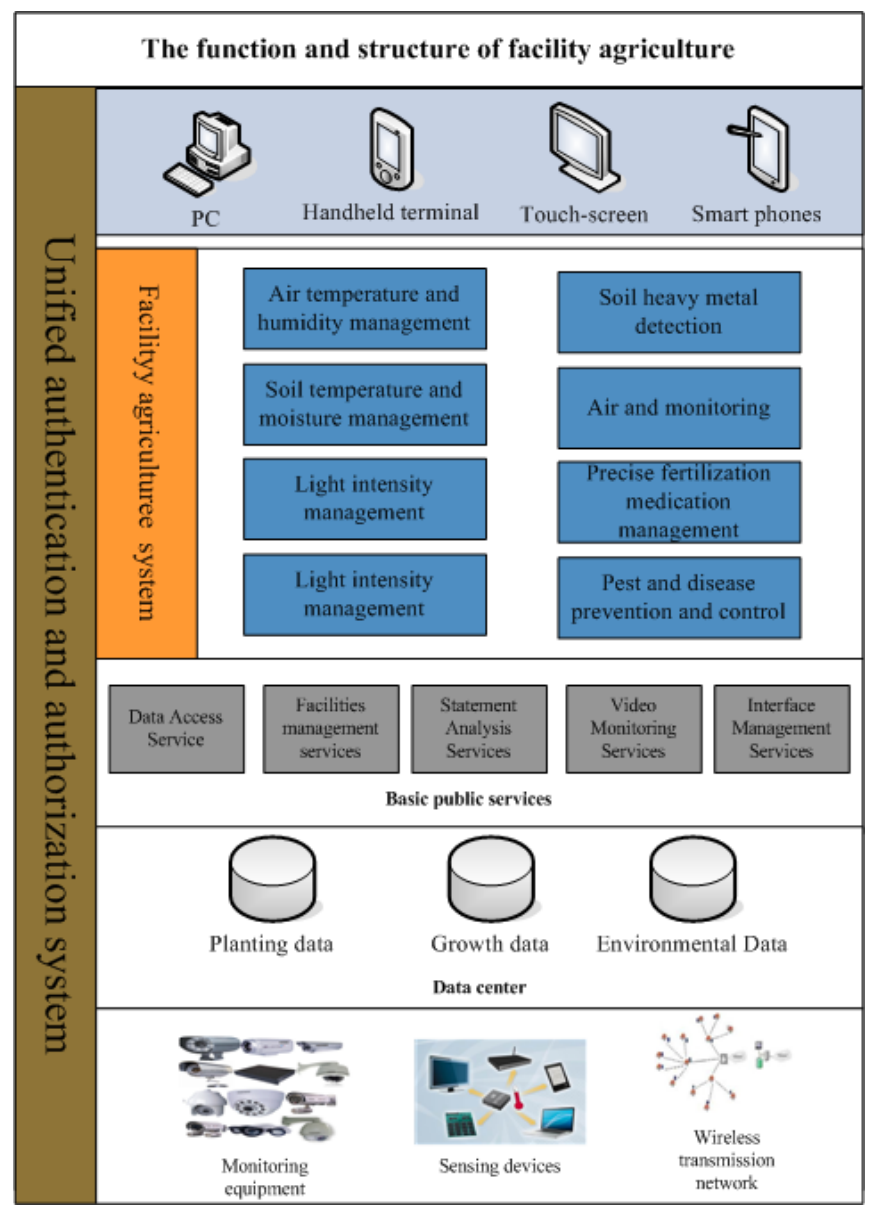

Fig. 2. The function and structure of facility agriculture

\subsection{Greenhouse Environment Monitoring System}

According to the facilities greenhouse cultivation of environmental conditions, we selected major environmental impact factor parameter detection. These parameters include meteorological parameters (light, temperature, humidity), environmental parameters ( $\mathrm{CO} 2$ and harmful gases such as ammonia, nitrous acid gas, $\mathrm{CO}$, etc.), soil parameters (temperature, humidity and $\mathrm{pH}, \mathrm{EC}$, soil composition).The environmental information within the greenhouse which gets by the integration of multiple sensors is sent to the smart processing platform by wireless sensor networks. It provides data support for agricultural decision-making. Greenhouse intelligent monitoring system is composed by the host computer, acquisition controller, network adapter, sensors, outdoor weather stations, and video capture equipment, and other components.

A host computer can connect multiple Acquisition Controllers and Acquisition Controllers connect the various sensors and control equipment to form a separate 
acquisition and control unit. Acquisition and control unit connected to the bus structure on the network communicate with the host computer independently. It controls the system remotely through the cable and the Internet way; regulates the temperature, light, ventilation, carbon dioxide supply, the supply and $\mathrm{pH}$ of the nutrient solution value, EC values.

Wireless sensor networks use hierarchical network structure, and a single greenhouse is a measurement of the wireless sensor network control area. Sensor nodes and control nodes in the network are self-organization; information is passed between nodes using multiple routes agreement. Data from all sensor nodes via a gateway to reach the control center, the feedback for greenhouse environment control information reaches the control node through the gateway. Control information controls valves, fans, temperature and other equipments to achieve the wireless monitoring and control of greenhouse environment. Fig.3 shows The greenhouses intelligent planting schematic.

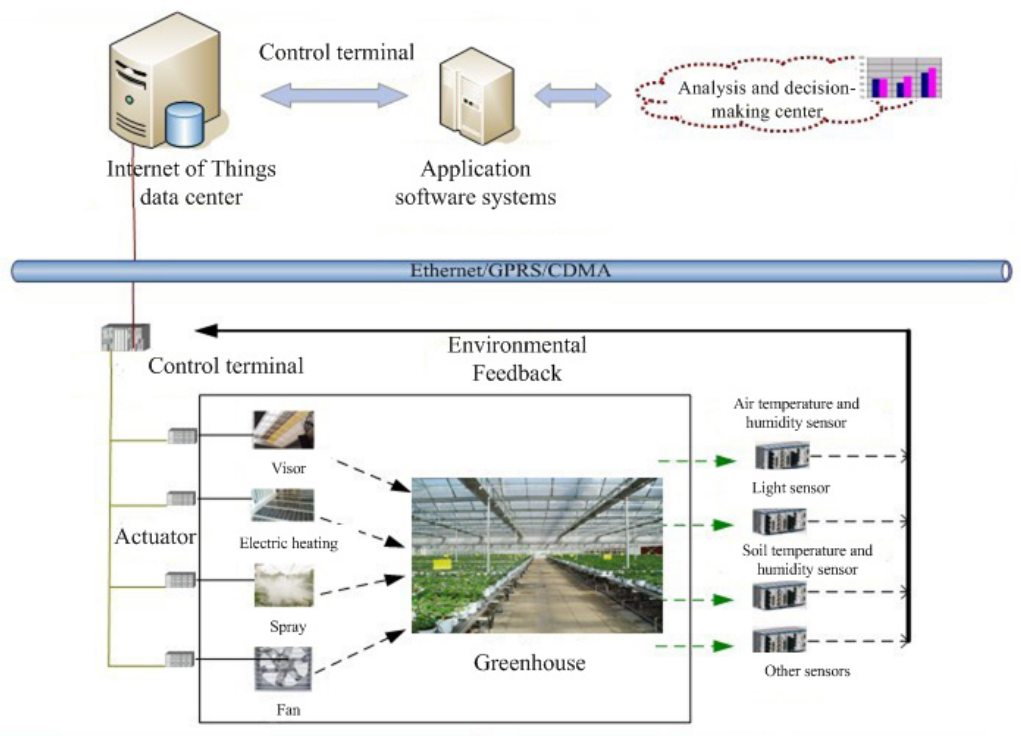

Fig. 3. The greenhouses intelligent planting schematic

\subsection{Digital Management System for Facility Agriculture}

Digital management technology analyzes intelligently the collected information; grasps all aspects of animal and plant growth status and makes decisions. The main contents are: monitoring, collection, processing and wireless transmission important environmental factors about vegetable growth; collecting data about facility vegetable growth; Modeling the growth simulation and optimization.

This system enables the query and distribution of agricultural information, the expert decision the Knowledge optimizes decision-making and analysis. It can achieve the efficient integration of information technology and network, and ultimate realize the agricultural precise digital control management. 


\section{Summary}

It is a trend to use information technology to lead the development of modern agriculture. The IntelliSense Internet of Things will be an important support for intensive, high-yield, high-quality, efficient, ecological security agriculture. Agricultural facilities can be time-out order-season production, improve land productivity, resource utilization and labor productivity. It has an important meaning for overcoming the bottleneck of resources and the environment of the agricultural development, transforming agricultural development, and enhancing the competitiveness of agriculture.

As a comprehensive application of technology in different disciplines, facility agricultural based on Internet of Things technology integrates a variety of technologies such as sensor, automation, communications, computer and animal plant sciences. It can be predicted that facility agricultural will have a rapid development in the promotion of agricultural machinery, sensors, information and communications and cloud computing technologies. It will play a major role to improve the overall efficiency of agriculture, promote the upgrade of modern agricultural transformation.

Acknowledgments. This study is financially supported by the National Key Technology R\&D Program of China (NO: 2012BAH20B02).

\section{References}

1. Collier, T.C., Kirschel, A., Taylor, C.E.: Acoustic localization of ant birds in a Mexican rainforest using a wireless sensor network. Journal of the Acoustical Society of America 128(1), 182-189 (2010)

2. Ignacio Huircan, J., Munoz, C., Young, H., et al.: ZigBee-based wireless sensor network localization for cattle monitoring in grazing fields. Computers and Electronics in Agriculture 74(2), 258-264 (2010)

3. Nadimi, E.S., Sogaard, H.T.: Observer Kalman filter identification and multiple-model adaptive estimation technique for classifying animal behaviour using wireless sensor networks. Computers and Electronics in Agriculture 68(1), 9-17 (2009)

4. Fukatsu, T., Hirafuji, M.: Field Monitoring Using Sensor Nodes with a Web Server. Journal of Robotics and Mechatronics 17(2), 164-172 (2005)

5. Nadimi, E.S., Sogaard, H.T., Bak, T., et al.: ZigBee-based wireless sensor networks for monitoring animal presence and pasture time in a strip of new grass. Computers and Electronics in Agriculture 61(2), 79-87 (2008)

6. Baggio, A.: Wireless Sensor Networks in Precision Agriculture. In: Proc. of the ACM Workshop on Real-World Wireless Sensor Networks, REAL WSN 2005 (2005)

7. Pierce, F.J., Elliott, T.V.: Regional and on-farm wireless sensor networks for agricultural systems in Eastern Washington. Computers and Electronics in Agriculture 61(1), $32-43$ (2008)

8. Lea-Cox, J.D., Ristvey, A.G., Arguedas, F.R., et al.: Wireless sensor networks for realtime management of irrigation and nutrient applications in the greenhouse and nursery industry. Hortscience 43(4), 1103 (2008)

9. Mizunuma, M., Katoh, T., Hata, S.: Applying IT to Farm Fields A Wireless LAN. NTT Techinical Review 1(2), 6-60 (2003)

10. Green, O., Nadimi, E.S., Blanes-Vidal, V., et al.: Monitoring and modeling temperature variations inside silage stacks using novel wireless sensor networks. Computers and Electronics in Agriculture 69(2), 149-157 (2009) 